\title{
Effective screening strategies for detection of asymptomatic COVID-19 travelers at airport quarantine stations: Exploratory findings in Japan
}

\author{
Masataro Norizuki ${ }^{1, *}$, Masahiko Hachiya ${ }^{1}$, Ayano Motohashi ${ }^{2}$, Ataru Moriya ${ }^{2}, K_{\text {Kazuhisa Mezaki }}{ }^{2}$ Moto Kimura $^{3}$, \\ Wataru Sugiura ${ }^{3}$, Hidechika Akashi ${ }^{1}$, Tamami Umeda ${ }^{1}$ \\ ${ }^{1}$ Bureau of International Health Cooperation, National Center for Global Health and Medicine, Tokyo, Japan; \\ ${ }^{2}$ Microbiology Laboratory, National Center for Global Health and Medicine, Tokyo, Japan; \\ ${ }^{3}$ Center for Clinical Sciences, National Center for Global Health and Medicine, Tokyo, Japan.
}

\begin{abstract}
The quantitative reverse transcription polymerase chain reaction method using nasopharyngeal swabs (NPS RT-qPCR) is regarded as the reference standard for diagnosing coronavirus disease 2019 (COVID-19). However, when using NPS RT-qPCR at busy airport quarantine stations, there are constraints on testing capacity, time, travelers' tolerance, and availability of personal protective equipment for quarantine officers. A feasible alternative is therefore needed to test incoming travelers, especially when passenger numbers increase with the resumption of business, tourism, and economic activities. To explore alternatives to NPS RT-qPCR, we collected nasopharyngeal, anterior nasal, and saliva samples chronologically over days 1-7 from asymptomatic COVID-19 air travelers who were under quarantine at a designated facility, and we then compared test results for 9 different methods, comprising RT-qPCR (including the reference method), loop-mediated isothermal amplification (LAMP), and qualitative and quantitative antigen testing. We evaluated sensitivity for 97 person-day samples independently to evaluate asymptomatic travelers regardless of their testing date and period of asymptomatic status upon entry. Sensitivity of the different tests varied from $46.6 \%$ to $81.0 \%$, but this was improved from $72.7 \%$ to $100.0 \%$ when the viral load was $>10^{4}$ copies/sample on NPS RT-qPCR. Thus, most high-risk asymptomatic travelers with higher viral load would be detected by the tests evaluated. Quantitative antigen testing using saliva samples showed $90.9 \%$ sensitivity and provided quicker results, and should be an acceptable alternative to NPS RT-qPCR at busy airport quarantine stations. We discuss the implications of our exploratory findings for establishing a comprehensive and feasible testing strategy for COVID-19 among air passengers.
\end{abstract}

Keywords: in vitro diagnostics, SARS-CoV-2, PCR, antigen testing, saliva, point of entry

\section{Introduction}

The global novel coronavirus disease 2019 (COVID-19) pandemic has led most countries to implement some form of travel restrictions, health screening and quarantine measures (1). In Japan, after COVID-19 was designated a quarantinable infectious disease on February 1, 2020 (2), quarantine officers started testing for symptomatic and suspected cases of COVID-19 infection. On March 9, entry restrictions became stricter, with quarantine measures strengthened to include testing even asymptomatic travelers by quantitative reverse transcription polymerase chain reaction (RT-qPCR) or loop-mediated isothermal amplification (LAMP) using nasopharyngeal swab samples (NPS), and isolation for COVID-19 positive travelers.

As asymptomatic carriers can unknowingly infect others, especially during the 2-3 days before symptom onset (3), all incoming travelers should be tested at the point of entry. Among the nucleic acid amplification testing (NAAT) methods available to use, NPS RT-qPCR is the reference standard. However, when conducted at quarantine stations, the results can take a long time, swab collection may cause travelers discomfort and bleeding, and quarantine officers are at risk of exposure, requiring personal protective equipment (PPE) to be worn. An alternative strategy is therefore needed for busy quarantine stations, particularly as economic and business activities resume and numbers of inbound travelers increase.

Several diagnostic tests for SARS-CoV-2 are approved for use in Japan (4), but there is no consensus or definitive guidance on the most effective method for mass screening of travelers. As all testing strategies have advantages and disadvantages, we felt it prudent to evaluate the feasibility of using alternatives to the reference standard NPS RT-qPCR. Therefore, in this study, we compared the sensitivity and feasibility of 
different tests using different samples against that of NPS RT-qPCR, by testing samples obtained from travelers confirmed positive for SARS-CoV-2 at airport quarantine. We report here our exploratory findings and suggest points to note when applying screening strategies with inbound travelers at airports.

\section{Sample collection and evaluation with 9 different testing methods}

Following approval by the Institutional Review Board of the National Center for Global Health and Medicine (NCGM-G-003641-00), we prospectively collected samples from inbound travelers to Japan who tested positive on NPS RT-qPCR or NPS LAMP at either of Tokyo's two international airports (Narita or Haneda) between July 27 and August 1, 2020. Of 7,689 passengers (from 283 commercial flights) whose samples were tested at the quarantine laboratory, 51 were positive for SARS-CoV-2. The 27 travelers diagnosed as COVID-19 asymptomatic carriers and transferred to a COVID-19 quarantine facility were eligible for this study (Figure S1, https://www.globalhealthmedicine.com/site/ supplementaldata.html? ID=17).

Full details of the inclusion criteria, sample collection, SARS-CoV-2 detection, and statistical analysis are provided as Supplementary Data (Methods S1, https:// www.globalhealthmedicine.com/site/supplementaldata. html?ID=17). Briefly, from day 1 until day 7 under quarantine, each participant was asked to provide a complete set of 4 self-collected samples and 2 physiciancollected samples (NPS) that were simultaneously obtained for testing on the same day. The self-collected samples were 2 saliva samples and 2 dry swab samples taken from the anterior $2 / 3$ of the dorsum of the tongue and the anterior nasal cavity. The 2 physician-collected samples were NPS samples, taken with a dry swab and a flocked swab. Samples were collected between July 27 to August 8, 2020, at which time the national quarantine measures changed. In that time frame, 20 quarantined individuals agreed to participate: most participants were male $(85 \%)$, age $<40$ years $(70 \%)$, most embarked in the Philippines (45\%), and $75 \%$ were seamen with special entry permission due to imminent departure from Japan by ship (Table 1). The number of participants who provided a complete set of samples every day was 4 for 7 days, 8 for 6 days, 2 for 5 days, 1 for 4 days, 1 for 3 days, and 4 for 1 day, yielding 97 person-day samples in total.

Samples were tested using the following 9 methods for detecting SARS-CoV-2 (Table S1, https://www. globalhealthmedicine.com/site/supplementaldata. html? ID =17) at independent facilities (see Methods $\mathrm{S} 1$ for details, https://www.globalhealthmedicine.com/ site/supplementaldata.html? ID=17), with sensitivity and $95 \%$ confidence intervals $(\mathrm{CI})$ calculated to assess the diagnostic performance of each test. Cohen's kappa (k) coefficients were then calculated to determine the
Table 1. Baseline characteristics of 20 international travelers diagnosed as asymptomatic carriers of COVID-19 at two airport quarantine stations in Tokyo (July 27-August 1, 2020)

\begin{tabular}{|c|c|c|}
\hline Variables & Number & $(\%)$ \\
\hline \multicolumn{3}{|l|}{ Sex } \\
\hline Male & 17 & $(85)$ \\
\hline Female & 3 & (15) \\
\hline \multicolumn{3}{|l|}{ Age, years } \\
\hline $20-29$ & 5 & $(25)$ \\
\hline $30-39$ & 9 & (45) \\
\hline $40-49$ & 5 & $(25)$ \\
\hline $50-59$ & 1 & (5) \\
\hline \multicolumn{3}{|l|}{ Country of embarkation } \\
\hline Philippines & 9 & $(45)$ \\
\hline India & 3 & (15) \\
\hline Bangladesh & 3 & (15) \\
\hline United Kingdom & 1 & (5) \\
\hline Mexico & 1 & (5) \\
\hline Pakistan & 1 & (5) \\
\hline France & 1 & (5) \\
\hline Ukraine & 1 & (5) \\
\hline \multicolumn{3}{|l|}{ Occupation } \\
\hline Seaman & 15 & $(75)$ \\
\hline Office worker & 2 & (10) \\
\hline Coordinator & 1 & $(5)$ \\
\hline Merchant seaman & 1 & (5) \\
\hline Unemployed & 1 & (5) \\
\hline \multicolumn{3}{|l|}{ Comorbidity } \\
\hline None & 20 & $(100)$ \\
\hline \multicolumn{3}{|l|}{ Smoking status } \\
\hline Current smoker & 2 & (10) \\
\hline Ex-smoker & 6 & (30) \\
\hline Never smoker & 12 & (60) \\
\hline \multicolumn{3}{|l|}{ Symptomatic status on entry ${ }^{a}$} \\
\hline Asymptomatic & 14 & $(70)$ \\
\hline Pre-symptomatic & 3 & (15) \\
\hline Post-symptomatic & 2 & (10) \\
\hline Pre- and post-symptomatic & 1 & (5) \\
\hline
\end{tabular}

${ }^{a}$ Symptomatic status was defined as follows: asymptomatic, asymptomatic before and upon entry to Japan; pre-symptomatic, developed symptoms during quarantine; post-symptomatic, symptoms had appeared before entry; and pre- and post-symptomatic, symptoms had appeared before and after entry.

concordance rate between the results of $i$ ) referencestandard NPS RT-qPCR and $i i)$ RT-qPCR using the anterior nasal swab sample (anterior nasal RT-qPCR); iii) RT-qPCR using a saliva sample (saliva RT-qPCR); $i v$ ) direct RT-qPCR using a saliva sample (saliva direct RTqPCR); v) LAMP using a saliva sample (saliva LAMP); $v i$ ) RT-qPCR using the tongue swab sample (tongue RTqPCR); vii) quantitative antigen testing using a saliva sample (quantitative saliva antigen); viii) quantitative antigen testing using an NPS sample (quantitative NPS antigen); and $i x$ ) qualitative rapid antigen testing using an NPS sample (qualitative NPS antigen; rapid antigen test). We used the cutoffs for quantitative antigen testing of 0.67 $\mathrm{pg} / \mathrm{mL}$ for saliva and $1 \mathrm{pg} / \mathrm{mL}$ for NPS, according to the manufacturer's instructions.

\section{Differences found between the various testing methods}

We evaluated sensitivity for the 97-person-day samples independently in order to evaluate the asymptomatic travelers regardless of their testing date and period of 
Table 2. Cohen's Kappa coefficients ( $\kappa$ ) and sensitivity for various tests compared with reference-standard NPS RT-qPCR (97 samples from 20 quarantined travelers diagnosed with COVID-19)

\begin{tabular}{|c|c|c|c|c|}
\hline \multirow{2}{*}{ Items } & \multirow{2}{*}{ Cohen's kappa } & \multicolumn{3}{|c|}{ Sensitivity (\%) } \\
\hline & & All & Viral load $\geq 10^{4}$ copies/sample & Viral load $<10^{4}$ copies/sample \\
\hline \multicolumn{5}{|l|}{ Self- collected samples } \\
\hline Anterior nasal RT-qPCR ${ }^{\mathrm{a}}$ & 0.56 & 69.0 & 100 & 28 \\
\hline Saliva RT-qPCR ${ }^{b}$ & 0.50 & 63.8 & 100 & 16 \\
\hline Saliva direct RT-qPCR ${ }^{c}$ & 0.39 & 46.6 & 81.8 & 0 \\
\hline Saliva LAMP ${ }^{\mathrm{d}}$ & 0.51 & 60.3 & 100 & 8 \\
\hline Tongue RT-qPCR ${ }^{\mathrm{a}}$ & 0.31 & 44.8 & 72.7 & 8 \\
\hline Quantitative saliva antigen testing ${ }^{\mathrm{e}}$ & 0.46 & 55.2 & 90.9 & 8 \\
\hline \multicolumn{5}{|l|}{ Physician-collected samples } \\
\hline Quantitative NPS antigen testing $^{\mathrm{e}}$ & 0.41 & 81.0 & 100 & 16 \\
\hline $\begin{array}{l}\text { Qualitative NPS antigen testing }^{\mathrm{f}} \\
\text { (rapid antigen test) }\end{array}$ & 0.55 & 60.3 & 100 & 8 \\
\hline
\end{tabular}

${ }^{\mathrm{a}} \operatorname{cobas}^{\circledR}$ SARS-CoV-2 (Roche Diagnostics, Indianapolis, IN). ${ }^{\mathrm{b}}$ Primer and probe set recommended by Japan's National Institute of Infectious Diseases $(13,14) .{ }^{c}$ SARS-CoV-2 Direct Detection RT-qPCR Kit (Takara Bio, Kusatsu, Japan). ${ }^{\mathrm{d}}$ Loopamp ${ }^{\circledR}$ 2019-SARS-CoV-2 Detection Reagent Kit (Eiken Chemical, Tokyo, Japan). ${ }^{\mathrm{e}}$ Lumipulse ${ }^{\circledR}$ G1200 system and Lumipulse SARS-CoV-2 Ag kit (Fujirebio, Tokyo, Japan). ${ }^{\mathrm{f}}$ ESPLINE SARSCoV-2 rapid antigen test (Fujirebio). NPS, nasopharyngeal swab; RT-qPCR, quantitative reverse transcription polymerase chain reaction.

asymptomatic status upon entry. Table 2 shows Cohen's kappa coefficients and sensitivity results for the 8 tests compared with NPS RT-qPCR. NAAT showed anterior nasal RT-qPCR had the highest sensitivity $(69 \%, 95 \%$ CI: 55.5-80.5), followed by saliva RT-qPCR (63.8\%, 50.1-76.0) and saliva LAMP (60.3\%, 46.6-73.0), with low sensitivity seen for saliva direct RT-qPCR $(46.6 \%$, 33.3-60.1) and tongue RT-qPCR (44.8\%, 31.7-58.5). Concordance between the tests was generally moderate but was low for tongue RT-qPCR $(0.31,0.13-0.49)$ and saliva direct RT-qPCR $(0.39,0.22-0.67)$. On quantitative NPS and saliva antigen testing, sensitivity was $55.2 \%$ (41.5-68.3) for saliva and 81.0\% (68.6-90.1) for NPS. On qualitative NPS antigen testing, sensitivity was $60.3 \%$ (46.6-73.0).

When viral load was $>10^{4}$ copies/sample for targets 1 and 2 on NPS RT-qPCR (33 samples), sensitivity was improved to $100 \%$ for anterior nasal RT-qPCR (95\% CI: 84.7-100), saliva RT-qPCR (84.7-100), quantitative NPS antigen (cut-off $\geq 1 \mathrm{pg} / \mathrm{mL}, 84.7-100$ ), saliva LAMP (84.7-100), and qualitative NPS antigen (84.7-100) and to $90.9 \%$ for quantitative saliva antigen (75.7-98.1), $81.8 \%$ for saliva direct RT-qPCR (64.5-93.0), and 72.7\% for tongue RT-qPCR (54.4-86.7). The detailed test results for all 20 participants are shown in Table S2 (https:// www.globalhealthmedicine.com/site/supplementaldata. html?ID=17).

\section{Possible implications of our exploratory findings}

Overall, some clear differences were evident between the 8 testing strategies compared with NPS RT-qPCR over days 1 to 7 , as determined by three independent laboratories, for asymptomatic international travelers who tested positive for SARS-CoV-2 on arrival at the airport. We have two major findings. First, compared with NPS RT-qPCR, the 8 tests showed varied sensitivity (44.8\%-81.0\%) and Cohen's kappa coefficients (0.31-
0.56), and the test results for tongue RT-qPCR, saliva direct RT-qPCR, and quantitative saliva antigen testing showed lower sensitivity $(44.8 \%, 46.6 \%$, and $55.2 \%$, respectively) than in previous studies (5-7). Second, the sensitivity of tongue RT-qPCR, saliva direct RTqPCR, saliva RT-qPCR, and quantitative saliva antigen testing was improved $(72.7 \%, 81.8 \%, 100 \%$, and $90.9 \%$, respectively) among participants showing a high viral load on NPS RT-qPCR.

Test sensitivity has varied across settings. NPS and saliva RT-qPCR showed highly consistent results in a mass-screening study in Japan of asymptomatic individuals from an airport quarantine group $(n=161)$ and a contact tracing group $(n=1,763)$; saliva RT-qPCR showed $92 \%$ sensitivity and $99.96 \%$ specificity (5). In American studies, estimated sensitivity was also high for tongue, nasal, and mid-turbinate RT-qPCR (89.8\%, $94.0 \%$, and $96.2 \%$, respectively) compared with NPS RT-qPCR (6), although saliva RT-qPCR showed around $30 \%$ lower sensitivity relative to NPS samples in a diagnostic cohort and around 50\% lower sensitivity in a convalescent cohort in a community setting (7). Possible reasons for saliva showing lower sensitivity in our study include the following. First, the results of saliva tests are more likely to be affected than NPS tests by unobserved self-collection of saliva samples and the oral cavity environment. Our participants self-collected samples unobserved while following instructions because we wanted to explore the feasibility of using this quick, easy, and well-tolerated saliva sampling method at busy airport quarantine stations. We asked participants to refrain from eating, drinking, chewing gum, and smoking for $1 \mathrm{~h}$ before saliva collection because these and similar activities may prevent SARS-CoV-2 detection in saliva (8). Thus, if saliva sampling is used at airports, cabin crew should remind passengers of these instructions well before landing. Also, self-collected saliva samples showed lower sensitivity than self-collected anterior 
nasal swabs, possibly because the latter were collected under observation. Second, the collection method, timing, storage, and processing of saliva samples is not standardized worldwide (9). Sensitivity may have been reduced with our collection methods compared with, for example, drooling into a tube or using a pipet. Third, we targeted asymptomatic passengers and collected samples over days 1 to 7 , which included participants found to be in the convalescent stage during the study period. Asymptomatic individuals were previously found to be less likely than symptomatic individuals to have detectable SARS-CoV-2 on NPS RT-qPCR (10), and our study did show lower sensitivity in the samples with lower viral load. Because asymptomatic carriers, in addition to symptomatic passengers, comprise the target population at airport quarantine stations, these four considerations should be kept in mind when evaluating the feasibility of saliva testing.

When evaluating the 9 different testing strategies for mass screening at busy airports, we considered sensitivity over time, speed, ease, and tolerability of sample collection. Saliva, tongue, and anterior nasal samples were, however, quicker and often more tolerable for travelers to provide than NPS samples (Table S1, https://www.globalhealthmedicine.com/ site/supplementaldata.html? ID=17). Antigen testing provided quicker results than NAAT (Table S1, https:// www.globalhealthmedicine.com/site/supplementaldata. html? ID=17), which is advantageous in a quarantine setting. We also evaluated self-collection of samples to reduce quarantine officers' exposure and PPE needs. For self-collected samples, saliva spit directly into a sterile tube showed more reliable results, and quarantine officers can visually confirm whether the sample is collected appropriately. Tongue and anterior nasal swabs are easy to self-collect, but quarantine officers should observe collection, so these methods are not suitable for busy quarantine stations.

From August 2020, based on a previous study (5), Japanese quarantine stations replaced NPS RT-qPCR or LAMP with quantitative saliva antigen testing for screening asymptomatic carriers (11). While we found that quantitative saliva antigen testing detected most asymptomatic carriers with higher viral load $(90.9 \%$ sensitivity), some travelers with lower viral load will test negative. When screening asymptomatic travelers, who have lower pretest probability (positive rate $0.66 \%$ in this study at airport), not all asymptomatic carriers will be detected by point of entry testing. Negative results can create a false sense of security, so quarantine officers could provide travelers with accurate information about testing, including limitations, and still encourage essential preventive measures.

For a comprehensive quarantine strategy, travelers who test positive on point of entry testing should naturally isolate, but also all negative travelers should routinely self-quarantine, avoid public transport, and undergo health monitoring for 14 days. To date, this has been successful, with surveillance systems in Japan having found no large clusters in the community involving inbound travelers.

\section{Using saliva samples in screening}

Quantitative saliva antigen testing showed 90.9\% sensitivity and provided relatively quick results, and should be an acceptable alternative to NPS RT-qPCR at busy airport quarantine stations. The points to note if using saliva samples to detect asymptomatic carriers are to $i$ ) remind passengers well before and upon landing to avoid eating, drinking, gargling, and smoking; $i$ ) give appropriate instructions for saliva collection in order to standardize procedures; and iii) develop systems for digitalized health monitoring, contact tracing, and healthcare consultations that respect inbound travelers' privacy, regardless of infection status.

\section{Future study}

In this exploratory study, we were not able to obtain definitive results about sensitivity and specificity. Also, we recruited only NPS RT-qPCR-positive travelers detected at airport quarantine stations, so we could not evaluate specificity for each testing procedure, as calculations for the specificity of each test should also include RT-qPCR-negative travelers. Other studies have also been relatively small so far, with 48 samples analyzed from 48 patients in a hospital setting (12) and 30 samples from 30 travelers quarantined with mild SARS-CoV-2 infection (10). We hope that reporting our exploratory findings here can inform the design of a larger multicenter study to examine feasible alternatives.

\section{Acknowledgments}

We thank Toyomitsu Tamura, Tomoko Komagata, Shikino Kikuchi, Yui Ito, and Toshiaki Baba at the National Center for Global Health and Medicine for assistance with testing and preparation for study, Hiroshi Ohtsu for advice on statistical analysis, and Caryn Jones of ThinkSCIENCE (Tokyo, Japan) for assistance with writing and editing the manuscript.

Funding: This study was supported by grants from the Ministry of Health, Labour and Welfare, Japan (Health, Labour and Welfare Policy Research Grant 20HA2002).

Conflicts of interest: The authors have no conflicts of interest to disclose.

\section{References}

1. Vaidya R, Herten-Crabb A, Spencer J, Moon S, Lillywhite 
L. Travel restrictions and infectious disease outbreaks. J Travel Med. 2020; 27:taaa050.

2. Ministry of Health, Labour and Welfare. Q\&A. March 26, 2020 version. https://www.mhlw.go.jp/stf/seisakunitsuite/ bunya/kenkou_iryou/covid19_qa_kanrenkigyou_00003. html (accessed February 4, 2021).

3. He X, Lau EHY, Wu P, et al. Temporal dynamics in viral shedding and transmissibility of COVID-19. Nat Med. 2020; 26:672-675.

4. Ministry of Health, Labour and Welfare. List of authorized in vitro diagnostics (IVDs) for COVID-19. https://www.mhlw.go.jp/stf/newpage_11332.html (accessed February 4, 2021).

5. Yokota I, Shane PY, Okada K, Unoki Y, Yang Y, Inao T, Sakamaki K, Iwasaki S, Hayasaka K, Sugita J, Nishida M, Fujisawa S, Teshima T. Mass screening of asymptomatic persons for SARS-CoV-2 using saliva. Clin Infect Dis. 2020. ciaa1388.

6. Tu YP, Jennings R, Hart B, Cangelosi GA, Wood RC, Webher K, Verma P, Vojta D, Berke EM. Swabs collected by patients or health care workers for SARS-CoV-2 testing. N Engl J Med. 2020; 383:494-496.

7. Becker D, Sandoval E, Amin A, De Hoff P, Diets A, Leonetti N, Lim YW, Elliot C, Laurent L, Grzymski J, Lu JT. Saliva is less sensitive than nasopharyngeal swabs for COVID-19 detection in the community setting. medRxiv. 2020. https://www.medrxiv.org/content/10.1101/2020.05.1 1.20092338v2 (accessed February 4, 2021).

8. Meister TL, Brüggemann Y, Todt D, Conzelmann C, Müller JA, Groß R, Münch J, Krawczyk A, Steinmann J, Steinmann J, Pfaender S, Steinmann E. Virucidal efficacy of different oral rinses against severe acute respiratory syndrome coronavirus 2. J Infect Dis. 2020; 222:12891292.

9. World Health Organization. Diagnostic testing for SARS-CoV-2: Interim guidance. https://apps.who.int/ iris/bitstream/handle/10665/334254/WHO-2019-nCoVlaboratory-2020.6-eng.pdf? sequence $=1 \&$ is Allowed $=y$ (accessed February 4, 2021).

10. Chau NVV, Thanh Lam V, Thanh Dung N, et al. The natural history and transmission potential of asymptomatic SARS-CoV-2 infection. Clin Infect Dis. 2020; 71:26792687.

11. Fujirebio. Japanese authorities have chosen Lumipulse ${ }^{\circledR}$ for nasopharyngeal or saliva-based SARS-CoV-2 antigen testing of passengers at their main international airports. https://www.fujirebio.com/en/insights/lung-diseases/ japanese-authorities-have-chosen-lumipulser-fornasopharyngeal-or (accessed February 4, 2021).

12. Wyllie AL, Fournier J, Casanovas-Massana A, et al. Saliva or nasopharyngeal swab specimens for detection of SARS-CoV-2. N Engl J Med. 2020; 383:1283-1286.

13. Shirato K, Nao N, Katano H, Takayama I, Saito S, Kato F, Katoh H, Sakata M, Nakatsu Y, Mori Y, Kageyama T, Matsuyama S, Takeda M. Development of genetic diagnostic methods for detection for novel coronavirus 2019(nCoV-2019) in Japan. Jpn J Infect Dis. 2020; 73:304-307.

14. National Institute for Infectious Diseases. Manual for the detection of pathogen 2019-nCoV ver.2.6. https://www.niid.go.jp/niid/images/epi/corona/2019nCoVmanual20200217-en.pdf (accessed February 4, 2021).

Received December 9, 2020; Revised February 5, 2021; Accepted February 26, 2021.

Released online in J-STAGE as advance publication March 9, 2021.

*Address correspondence to:

Masataro Norizuki, Bureau of International Health Cooperation, National Center for Global Health and Medicine, 1-21-1 Toyama, Shinjuku, Tokyo 162-8655, Japan.

E-mail: m-norizuki@it.ncgm.go.jp 